\title{
Multiobjective Multicast Routing with Ant Colony Optimization
}

\author{
Diego Pinto and Benjamín Barán \\ Polytechnical School, National University of Asunción \\ P.O. Box CC 2111 - Paraguay \\ \{dpinto,bbaran\}@pol.una.py \\ http://www.fpuna.edu.py/
}

\begin{abstract}
This work presents a multiobjective algorithm for multicast traffic engineering. The proposed algorithm is a new version of MultiObjective Ant Colony System (MOACS), based on Ant Colony Optimization (ACO). The proposed MOACS simultaneously optimizes the maximum link utilization, the cost of the multicast tree, the averages delay and the maximum end-to-end delay. In this way, a set of optimal solutions, known as Pareto set is calculated in only one run of the algorithm, without a priori restrictions. Experimental results obtained with the proposed MOACS were compared to a recently published Multiobjective Multicast Algorithm (MMA), showing a promising performance advantage for multicast traffic engineering.
\end{abstract}

\section{Introduction}

Multicast consists of simultaneous data transmission from a source node to a subset of destination nodes in a computer network [1]. Multicast routing algorithms have recently received great attention due to the increased use of recent point-tomultipoint applications, such as radio and TV transmission, on-demand video, teleconferences and so on. Such applications generally require optimization of several quality-of service $(Q o S)$ parameters such as maximum end-to-end delay and minimum use of bandwidth resources in a context of traffic engineering.

When a dynamic multicast problem considers several traffic requests, not only QoS parameters must be considered, but also load balancing and network resources must be taken into account. In order to avoid hot spots and to balance the network load, a common approach is to minimize the utilization of the most heavily used link in the network $(\alpha)$ or maximum link utilization [2]. At the same time, cost minimization of the tree of each multicast group, which is given by the sum of the cost of the used links, is also desired. It is known that the complexity of computing the minimum cost tree for a given multicast group is NP-hard [3]. Then, this paper presents a new MultiObjective Ant Optimization System (MOACS) [4], which finds a set of optimal solutions by simultaneously minimizing the maximum link 
utilization, the cost of the tree, the maximum end-to-end delay and the average delay. In this way, a whole Pareto set of optimal solutions can be obtained in only one run of the proposed algorithm.

The remainder of this paper is organized as follows. Section 2 describes related works. A general definition of an Optimization Multiobjective Problem is presented in Section 3. The problem formulation and the objective functions are given in Section 4. The proposed algorithm is explained in Section 5 while a brief description of MMA algorithm is given in Section 6. The experimental environment is shown in Section 7 and experimental results are present in Section 8. Finally, conclusions and future works are presented in Section 9.

\section{Related work}

Several algorithms based on ACO consider multicast routing as a mono-objective problem, minimizing the cost of the tree under multiple constrains. In [5] Liu and $\mathrm{Wu}$ proposed the construction of a multicast tree, where only the cost of the tree is minimized using degree constrains. On the other hand, Gu et al. considered multiple parameters of QoS as constrains, minimizing just the cost of the tree [6]. It can be clearly noticed that previous algorithms treated the Traffic Engineering Multicast problem as a mono-objective problem with several constrains. The main disadvantage of this approach is the necessity of an a priori predefined upper bound that can exclude good trees from the final solution. In [3], Donoso et al. proposed a multi-tree traffic-engineering scheme using multiple trees for each multicast group. They took into account four metrics: (i) maximum link utilization $\alpha$, (ii) hop count, (iii) bandwidth consumption and (iv) total end-to-end delay. The method minimizes a weighted sum function composed of the above four metrics. Considering the problem is NP-hard, the authors proposed a heuristic algorithm consisting of two steps: (1) obtaining a modified graph, where all possible paths between the source node and every destination node are looked for, and (2) finding out the trees based on the distance values and the available capacity of the paths, in the modified graph. Crichigno and Barán [7] have proposed a Multiobjective Multicast Algorithm (MMA), based on the Strength Pareto Evolutionary Algorithm (SPEA) [8], which simultaneously optimizes the maximum link utilization, the cost of the tree, the maximum end-to-end delay and the average delay. This MMA algorithm finds a set of optimal solutions, which is calculated in only one run, without a priori restrictions.

\section{Multiobjective Optimization Problem}

A general Multiobjective Optimization Problem (MOP) [9] includes a set of $n$ decision variables, $k$ objective functions, and $m$ restrictions. Objective functions and restrictions are functions of decision variables. This can be expressed as:

$$
\begin{array}{ll}
\text { Optimize } & \boldsymbol{y}=f(\boldsymbol{x})=\left(f_{l}(\boldsymbol{x}), f_{2}(\boldsymbol{x}) \ldots, f_{k}(\boldsymbol{x})\right) . \\
\text { Subject to } & e(\boldsymbol{x})=\left(e_{l}(\boldsymbol{x}), e_{2}(\boldsymbol{x}), \ldots, e_{m}(\boldsymbol{x})\right) \geq \mathbf{0}, \\
\text { where } & \boldsymbol{x}=\left(x_{l}, x_{2}, \ldots, x_{n}\right) \in \boldsymbol{X} \text { is the decision vector, } \\
\text { and } & \boldsymbol{y}=\left(y_{1}, y_{2}, \ldots, y_{k}\right) \in \boldsymbol{Y} \text { is the objective vector. }
\end{array}
$$


$\boldsymbol{X}$ denotes the decision space while the objective space is denoted by $\boldsymbol{Y}$. Depending on the kind of the problem, "optimize" could mean minimize or maximize. The set of restrictions $e(x) \geq 0$ determines the set of feasible solutions $X_{f} \subseteq X$ and its corresponding set of objective vectors $Y_{f} \subseteq Y$. The problem consists in finding $x$ that optimizes $f(x)$. In general, there is no unique "best" solution but a set of solutions, none of which can be considered better than the others when all objectives are considered at the same time. This derives from the fact that there can be conflicting objectives. Thus, a new concept of optimality should be established for MOPs. Given two decision vectors $u, v \in X_{f}$ :

$$
\begin{array}{lll}
f(u)=f(v) & \text { iff: } & \forall i \in\{1,2, \ldots, \mathrm{k}\}: f_{i}(u)=f_{i}(v) \\
f(u) \leq f(v) & \text { iff: } & \forall i \in\{1,2, \ldots, \mathrm{k}\}: f_{i}(u) \leq f_{i}(v) \\
f(u)<f(v) & \text { iff: } & f(u) \leq f(v) \wedge f(u) \neq f(v)
\end{array}
$$

Then, in a minimization context, $u$ and $v$ comply with one and only one of the following three possible conditions:

$$
\begin{aligned}
& u>v(u \text { dominates } v), \text { iff: } f(u)<f(v) \\
& v>u(v \text { dominates } u), \text { iff: } f(v)<f(u) \\
& u \sim v(u \text { and } v \text { are non-comparable), iff: } f(u) \nless f(v) \wedge f(v) \nless f(u)
\end{aligned}
$$

Alternatively, for the rest of this work, $u \triangleleft v$ will denote that $u \succ v$ or $\boldsymbol{u} \sim \boldsymbol{v}$. A decision vector $x \in X_{f}$ is non-dominated with respect to a set $Q \subseteq X_{f}$ iff: $x \triangleleft v, \forall v \in Q$. When $x$ is non-dominated with respect to the whole set $X_{f}$, it is called an optimal Pareto solution; therefore, the Optimal Pareto set $\boldsymbol{X}_{\text {true }}$ may be formally defined as:

$$
X_{t r u e}=\left\{x \in X_{f} \mid x \text { is non-dominated with respect to } X_{f}\right\}
$$

The corresponding set of objective vectors $Y_{\text {true }}=f\left(X_{\text {true }}\right)$ constitutes the Optimal Pareto Front.

\section{Problem Formulations}

For this work, a network is modeled as a direct graph $G=(V, E)$, where $V$ is the set of nodes and $E$ is the set of links. Let:

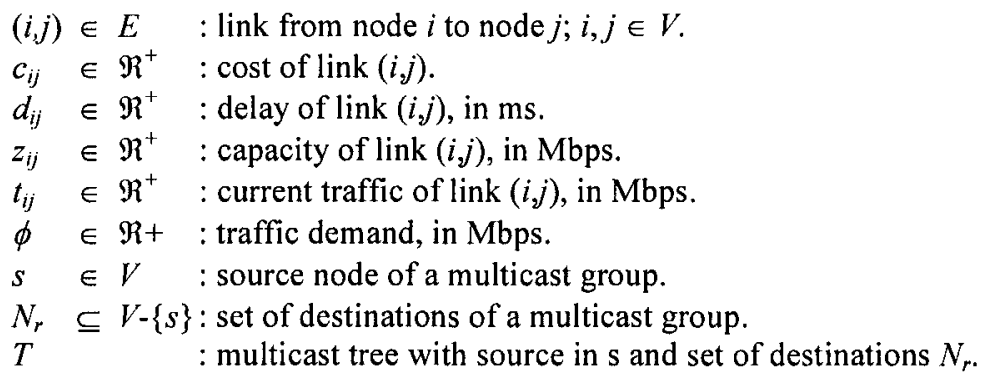


Also, let $p(s, n) \subseteq T$ be the path that connects the source node $s$ with a destination node $n \in N_{r}$. Finally, let $d_{p(s, n)}$ represent the delay of the path $p(s, n)$, given by the sum of the link delays that conform the path, i.e.:

$$
d_{p(s, n)}=\sum_{(i, j) \in p(s, n)} d_{i j}
$$

Using the above definitions, a multicast routing problem for Traffic Engineering may be stated as a MOP that tries to find the multicast tree $T$ that simultaneously minimizes the following objective functions:

1- Maximum link utilization of the tree:

$$
\alpha_{m}=\underset{(i, j) \in T}{\operatorname{Max}}\left\{\alpha_{i j}\right\}
$$

where $\alpha_{i j}=\left(\phi+t_{i j}\right) / z_{i j}$.

2- Cost of the multicast tree:

$$
C=\phi * \sum_{(i, j) \in T} c_{i j}
$$

3- Maximum end-to-end delay of the multicast tree:

$$
D_{m}=\underset{n \in N_{r}}{\operatorname{Max}}\left\{d_{p(s, n)}\right\}
$$

4-Average delay of the multicast tree:

$$
D_{a}=\frac{1}{\left|N_{r}\right|} * \sum_{n \in N_{r}} d_{p(s, n)}
$$

where $\left|N_{r}\right|$ denotes the cardinality of $N_{r}$.

The problem is subject to a link capacity constraint:

$$
\alpha_{i j} \leq 1 \quad \forall(i, j) \in T
$$

A simple example follows to clarify the above notation.

Example 1. Given the NSF network topology of Fig. 1 [7], the number over each link $(i, j)$ denotes $d_{i j}$ in ms, $c_{i j}$, and $t_{i j}$ at a given time (in Mbps). NSF network consist of 14 nodes and for each link, $z_{i j}=1.5 \mathrm{Mbps}$. Let suppose a traffic request arriving with $\phi=0.2 \mathrm{Mbps}, s=5$, and $N_{r}=\{0,2,6,13\}$. Fig. 1 shows a multicast tree $(T)$ while Table 1 presents the objective functions calculated for this tree.

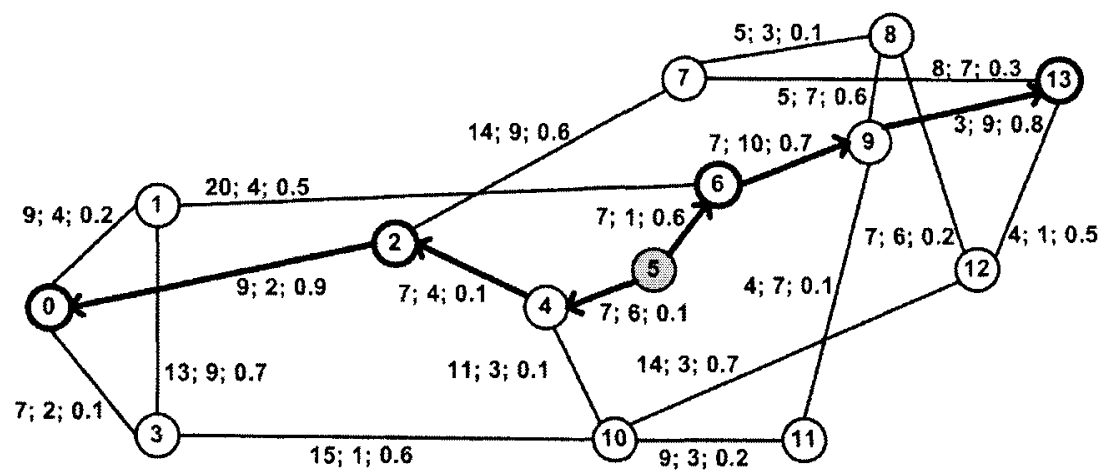

Fig. 1. The NSF Net. $\alpha_{m}=0.73 ; C=6.4 ; D_{m}=23 ; D_{a}=16.5$. 
Table 1. Objective Functions Calculated for Example 1.

\begin{tabular}{|c|c|c|c|c|c|c|}
\hline \multicolumn{7}{|c|}{ Tree } \\
\hline$(i, j)$ & $(5,4)$ & $(4,2)$ & $(2,0)$ & $(5,6)$ & $(6,9)$ & $(9,13)$ \\
\hline$d_{i j}$ & 7 & 7 & 9 & 7 & 7 & 8 \\
\hline$c_{i j}$ & 6 & 4 & 2 & 1 & 10 & 9 \\
\hline$t_{i j}$ & 0.1 & 0.1 & 0.9 & 0.6 & 0.7 & 0.8 \\
\hline$z_{i j}$ & 1.5 & 1.5 & 1.5 & 1.5 & 1.5 & 1.5 \\
\hline$\alpha_{i j}$ & 0.2 & 0.2 & 0.73 & 0.53 & 0.6 & 0.53 \\
\hline \multicolumn{7}{|c|}{ Delay paths } \\
\hline $\begin{array}{l}d_{p(5,2)} \\
d_{p(5,0)} \\
d_{p(5,6)} \\
d_{p(5,13)}\end{array}$ & \multicolumn{6}{|c|}{$\begin{aligned} d_{5,4}+d_{4,2}=7+7 & =14 \\
d_{5,4}+d_{4,2}+d_{2,0}=7+7+9 & =23 \\
d_{5,6} & =7 \\
d_{5,6}+d_{6,9}+d_{9,13}=7+7+8 & =22\end{aligned}$} \\
\hline \multicolumn{7}{|c|}{ Metrics of the solution Tree } \\
\hline $\begin{array}{l}\alpha_{m} \\
C \\
D_{m} \\
D_{a}\end{array}$ & \multicolumn{6}{|c|}{$\begin{aligned} \alpha_{2,0} & =\mathbf{0 . 7 3} \\
\phi^{*}\left(c_{5,4}+c_{4,2}+c_{2,0}+c_{5,6}+c_{6,9}+c_{9,13}\right)=0.2 *(6+4+2+1 & +10+9) \\
& =\mathbf{6 . 4} \\
& =\mathbf{2 3} \\
d_{p(5,0)} & =\mathbf{6 . 5}\end{aligned}$} \\
\hline
\end{tabular}

For the same example, Figure 2 presents in (a), (b) and (c) three different alternative solutions, for the same multicast group, to clarify the concept of nondominance. Notice that each tree is better than any other in at least one objective.

It is important to notice, from the mathematical formulation that the four objective functions are treated independently and should be minimized simultaneously. They are not combined to form a scalar single-objective function through a linear combination (as weighted sum) nor are any of them treated as a restriction. This way, using the concept of dominance, a whole set of optimal Pareto solutions is calculated.

Table 2. "Optimal Pareto Set" and "Optimal Pareto Front" for Example 1.

\begin{tabular}{|c|c|c|c|c|c|}
\hline & \multirow{2}{*}{$\begin{array}{c}\text { Optimal Pareto Set } \\
\text { (Trees) }\end{array}$} & \multicolumn{4}{|c|}{$\begin{array}{l}\text { Optimal Pareto Front } \\
\text { (Objective Vectors) }\end{array}$} \\
\hline & & $\alpha_{m}$ & $C$ & $D_{m}$ & $D_{a}$ \\
\hline$\overline{S_{l}}$ & $(5,6),(5,4),(4,2),(4,10),(2,0),(10,12),(12,13)$ & 0.73 & 5 & 20 & 36 \\
\hline $\boldsymbol{S}_{2}$ & $(5,6),(5,4),(6,1),(6,9),(4,2),(1,0),(9,8),(8,12),(12,13)$ & 0.6 & 8.6 & 21.75 & 36 \\
\hline$S_{3}$ & $(5,6),(5,4),(6,1),(6,9),(4,2),(1,0),(9,13)$ & 0.67 & 7.6 & 19.75 & 36 \\
\hline$S_{4}$ & $(5,6),(5,4),(6,9),(4,2),(9,13),(2,0)$ & 0.73 & 6.4 & 16.50 & 23 \\
\hline$S_{5}$ & $(5,6),(5,4),(6,1),(4,2),(4,10),(1,0),(10,12),(12,13)$ & 0.73 & 4 & 26.75 & 63 \\
\hline$S_{6}$ & $(5,6),(5,4),(6,1),(4,2),(4,10),(1,0),(10,12),(12,13)$ & 0.6 & 6.2 & 23.25 & 36 \\
\hline$S_{7}$ & $(5,6),(6,1),(1,0),(0,3),(0,2),(3,10),(10,12),(12,13)$ & 0.73 & 3.6 & 41 & 76 \\
\hline$S_{8}$ & $(5,6),(5,4),(6,1),(4,2),(1,0),(2,7),(7,13)$ & 0.53 & 7 & 23.75 & 38 \\
\hline$S_{g}$ & $(5,6),(5,4),(4,2),(4,10),(10,12),(10,3),(12,13),(3,0)$ & 0.6 & 5.2 & 24.25 & 4 \\
\hline$\underline{S}_{10}$ & $(5,6),(5,4),(4,10),(10,3),(10,12),(3,0),(12,13),(0,2)$ & 0.73 & 4.8 & 33 & 49 \\
\hline
\end{tabular}




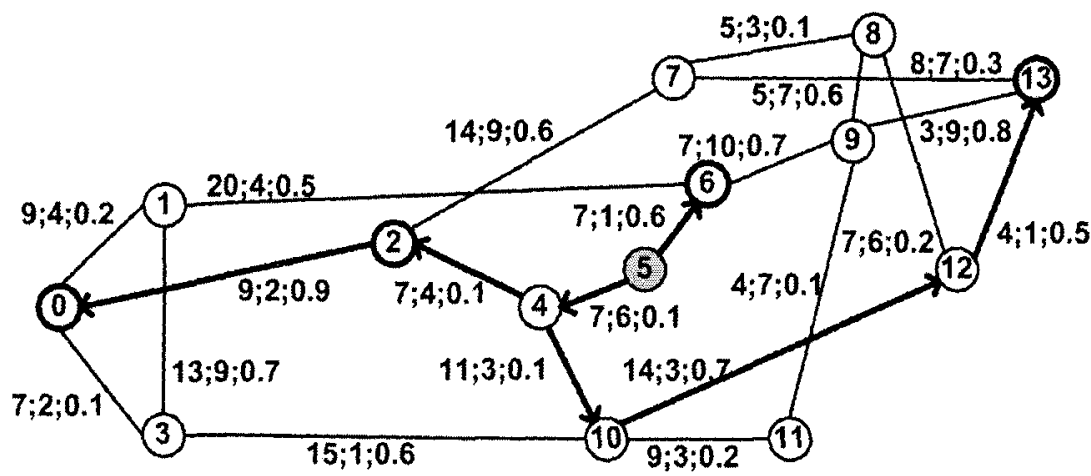

(a)

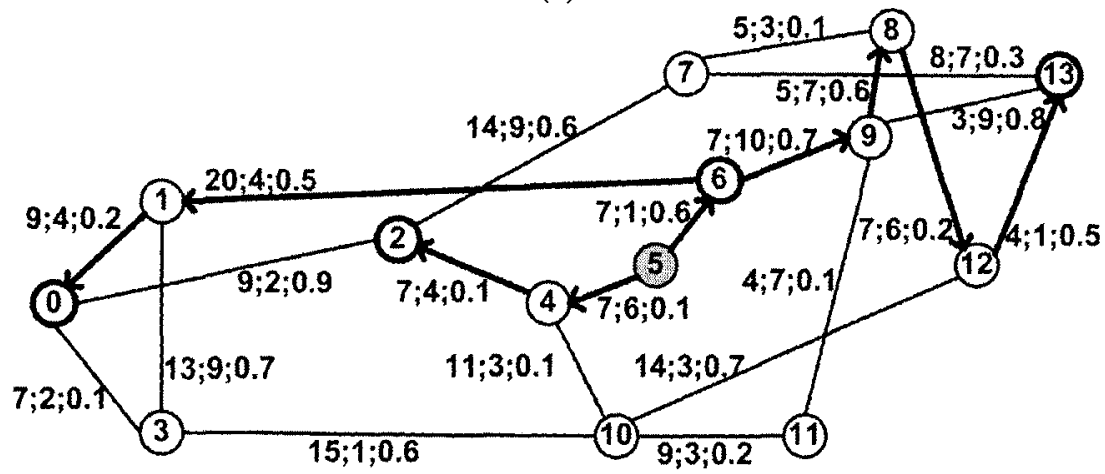

(b)

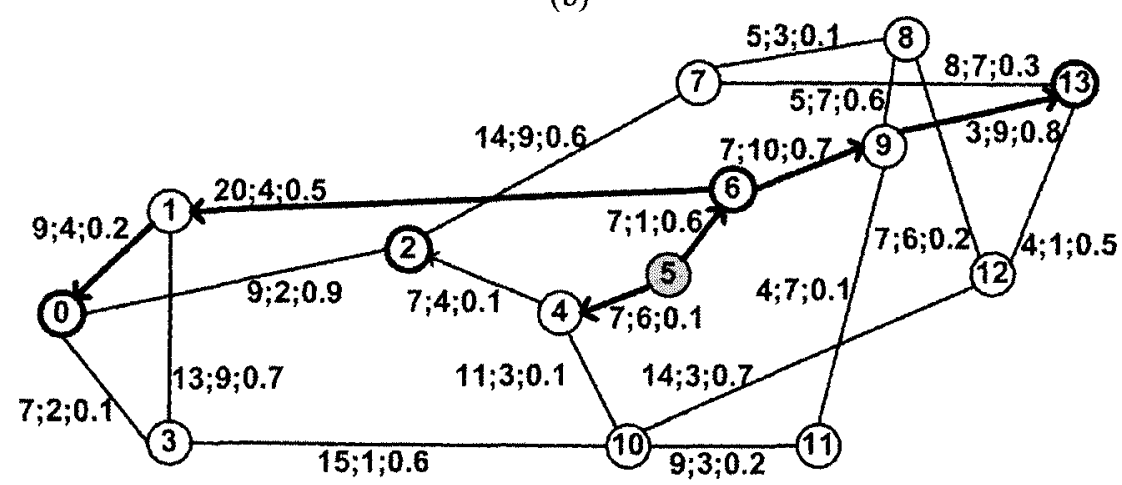

(c)

Fig. 2. The NSF Net. (a) to (c) show different Pareto solutions for the same multicast group of example 1 .

For the presented example, the set of optimal Pareto set and corresponding objective functions are shown in Table 2 . Notice that solution $\mathrm{S}_{1}$ corresponds to Figure 2(a), $S_{2}$ corresponds to Figure $2(b)$ and $S_{3}$ corresponds to Figure 3(c). 


\section{Ant Colony Optimization Approach}

Ant Colony Optimization (ACO) is a metaheuristic inspired by the behavior of natural ant colonies [10]. In the last few years, ACO has received increased attention by the scientific community as can be seen by the growing number of publications and different application fields [4]. Even though, there are several ACO variants that can be considered, a standard approach is next presented [11].

\subsection{Standard Approach}

ACO uses a pheromone matrix $\tau=\left\{\tau_{i j}\right\}$ for the construction of potential good solutions. The initial values of $\tau$ are set as $\tau_{i j}=\tau_{0} \forall(i, j)$, where $\tau_{0}>0$. It also takes advantage of heuristic information (known as visibility) using $\eta_{i j}=1 / d_{i j}$. Parameters $\alpha$ and $\beta$ define the relative influence between the heuristic information and the pheromone levels [10]. While visiting node $i, N_{i}$ represents the set of neighbor nodes that are not yet visited. The probability $\left(p_{i j}\right)$ of choosing a next node $j$, while visiting node $i$, is defined by equation (11). At every iteration of the algorithm, each ant of a colony constructs a complete solution $T$ using (11), starting at source node $s$. Pheromone evaporation is applied for all $(i, j)$ of $\tau$, according to $\tau_{i j}=(1-\rho) \cdot \tau_{i j}$, where parameter $\rho \in(0 ; 1]$ determines the evaporation rate. Considering an elitist strategy, the best solution found so far $T_{b e s t}$ updates $\tau$ according to $\tau_{i j}=\tau_{i j}+\Delta \tau$, where $\Delta \tau=1 / l\left(T_{\text {best }}\right)$ if $(i, j) \in T_{\text {best }}$ and $\Delta \tau=0$ otherwise. Where $l\left(T_{\text {best }}\right)$ represents and objective function to be minimized.

$$
p_{i j}= \begin{cases}\frac{\tau_{i j}^{\alpha} \cdot \eta_{i j}^{\beta}}{\sum_{g \in N_{i}} \tau_{i g}^{\alpha} \cdot \eta_{i g}^{\beta}} & \text { if } j \in N_{i} \\ 0 & \text { otherwise }\end{cases}
$$

\subsection{Proposed Algorithm}

Following the MultiObjective Ant Colony Optimization Algorithm (MOACS) scheme [4], which is a generalization of the ACS [10], the proposed algorithm uses a colony of ants (or agents) for the construction of $m$ solutions $T$ at every generation. Then, a known Pareto Front $Y_{\text {know }}$ [9] is updated including the best non-dominate solutions that have been calculated so far. Finally, the gathered information is saved updating a pheromone matrix $\tau_{i j}$. Fig. 3 (a) presents the general procedure of MOACS. In general, if the state of $Y_{\text {know }}$ was changed, the pheromone matrix $\tau_{\mathrm{ij}}$ is re-initialized $\left(\tau_{\mathrm{ij}}=\tau_{0} \forall(i, j) \in V\right)$ to improve exploration in the decision space $X_{f}$. Otherwise, $\tau_{\mathrm{ij}}$ is globally updated using the solutions of $Y_{\text {know }}$ to exploit the knowledge of the best known solutions. Note that only the links of found solutions $T$ in $Y_{\text {know }}$ are used to update the pheromone matrix $\tau_{i j}$. To construct a solution, an ant begins its job in the source node $s$. A non-visited node is pseudo-randomly [4] selected at each step. This process continues until all destination nodes of the multicast group are reached. Considering $R$ as the list of starting nodes, $K_{i}$ as the list of feasible neighboring nodes to the node $i, D_{r}$ as the set of destination nodes already reached, the procedure to find a solution $T$ is summarized in Fig. 3 (b). 


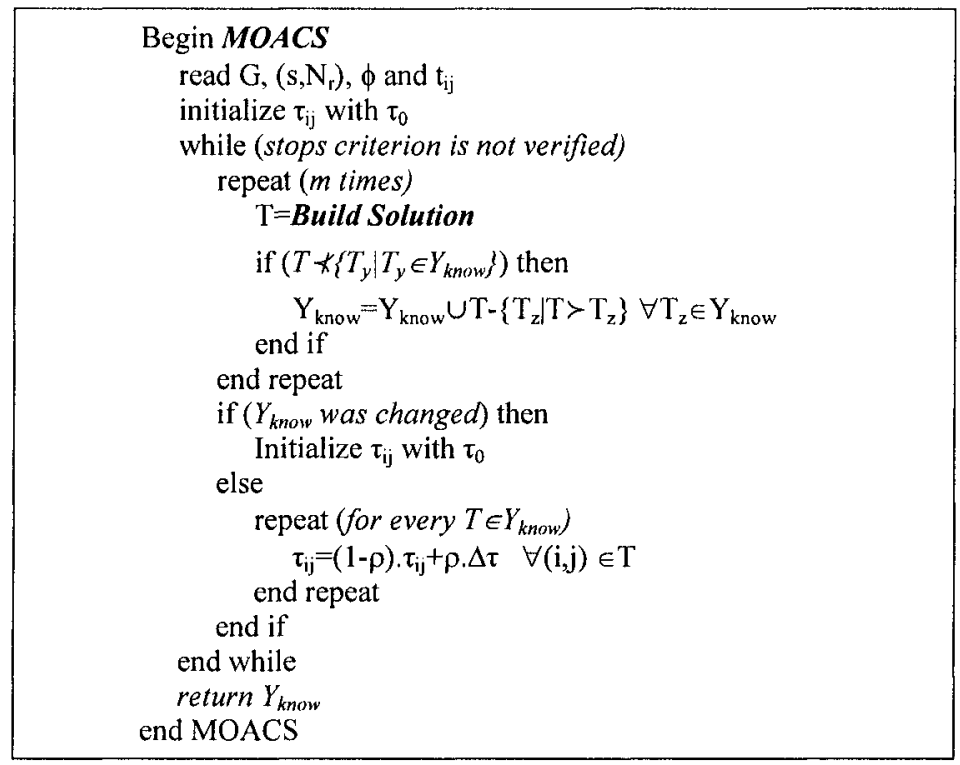

\section{(a)}

$$
\begin{aligned}
& \text { Begin Build Solution } \\
& \mathrm{T}=\{\varnothing\} ; \mathrm{D}_{\mathrm{r}}=\{\varnothing\} ; \mathrm{R}=\mathrm{R} \cup \mathrm{s} \\
& \text { repeat (until } R=\{\varnothing\} \text { or } D_{r}=N_{r} \text { ) } \\
& \text { select node } \mathrm{i} \text { of } \mathrm{R} \text { and build set } \mathrm{K}_{\mathrm{i}} \\
& \text { if }\left(K_{i}=\{\varnothing\}\right) \text { then } \\
& \mathrm{R}=\mathrm{R}-\mathrm{i} / \text { /*erase node without feasible neighbor*/ }
\end{aligned}
$$

Fig. 3. (a) General Procedure of MOACS and (b) Procedure to Build Solution. where:

$$
\Delta \tau=\frac{1}{\sum_{\forall T \in \xi_{\text {tam }}}\left(\alpha_{m} * C * D_{m} * D_{a}\right)}
$$

and $\rho \in(0,1]$ represents trail persistence. 


\section{Multiobjective Multicast Algorithm}

Multiobjective Multicast Algorithm (MMA), recently proposed in [7], is based on the Strength Pareto Evolutionary Algorithm (SPEA) [8]. MMA holds an evolutionary population $P$ and an external Pareto solution set $P_{n d}$. Starting with a random population $P$ of solutions, the individuals evolve to Pareto optimal solutions to be included in $P_{n d}$. The pseudo-code of the main MMA algorithm is shown in Fig. 4(a), while its codification is represented in Fig. 4(b).

\begin{tabular}{|l|}
\hline Begin $M M A$ \\
Read $\mathrm{G},\left(\mathrm{s}, \mathrm{N}_{\mathrm{r}}\right), \phi$ and $\mathrm{t}_{\mathrm{ij}}$ \\
Build routing tables \\
Initialize $P$ \\
Do \{ \\
Discard individuals \\
Evaluate individuals \\
Update non-dominated set $P_{n d}$ \\
Compute fitness \\
Selection \\
Crossover and Mutation \\
while stop criterion is not verified \\
end MMA
\end{tabular}

(a)

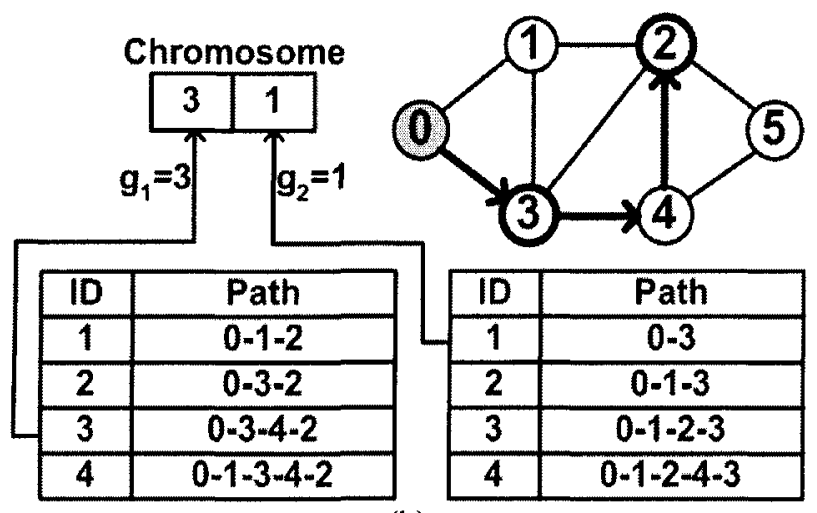

(b)

Fig. 4. (a) Pseudo-code of main MMA algorithm (b) Relationship between a chromosome, genes and routing tables for a tree with $s=0$ and $N_{r}=\{2,3\}$.

The MMA algorithm begins reading the variables of the problem and basically proceeds as follows (see pseudo-code in Fig. 4(a)):

Build routing tables: For each $n_{i} \in N_{r}$, a routing table is built. It consists of the $\psi$ shortest and $\psi$ cheapest paths. $\psi$ is a parameter of the algorithm. A chromosome is represented by a string of length $\left|N_{r}\right|$ in which each element (gene) $g_{i}$ represents a 
path between $s$ and $n_{i}$. See Fig. 4(b) to see a chromosome that represents the tree in Fig. 4(b).

Discard individuals: In $P$, there may be duplicated chromosomes. Thus, new randomly generated individuals replace duplicated chromosomes.

Evaluate individuals: The individuals of $P$ are evaluated using the objective functions. Then, non-dominated individuals of $P$ are compared with the individuals in $P_{n d}$ to update the non-dominated set, removing from $P_{n d}$ dominated individuals.

Compute fitness: Fitness is computed for each individual, using SPEA procedure [8].

Selection: Traditional tournament or roulette methods may be used [8]. In this works, a roulette selection operator is applied over the set $P_{n d} \cup P$ to generate the next evolutionary population $P$.

Crossover and Mutation: MMA uses two-point crossover operator over selected pair of individuals. Then, some genes in each chromosome of the new population are randomly changed (mutated), obtaining a new solution. The process continues until a stop criterion, as a maximum number of generations, is satisfied.

\section{Experimental Environments}

MOACS and MMA have been implemented on a $350 \mathrm{MHz}$ AMD Athlon computer with a $128 \mathrm{MB}$ of RAM. The compiler used was Borland C++ V 5.02.

In order to evaluate the proposed MOACS approach to a recently published algorithm as MMA [7], several test problems were used, but only two will be presented. Each test was divided into four sub-tests (or scenarios) where the networks are under different load level:

- low $\quad\left(0 \leq \alpha_{i j} \leq 0.4\right)$,

- medium $\left(0.4 \leq \alpha_{i j} \leq 0.7\right)$,

- high $\left(0.7 \leq \alpha_{i j} \leq 0.9\right)$, and

- saturation $\left(0.9 \leq \alpha_{i j} \leq 1\right)$.

MMA parameters were: 40 chromosomes and mutation probability of 0.3 , suggested in [7], while MOACS parameters were: 40 ant, 0.95 pseudo-random probability, 0.95 trail persistence. The runs stopped after 2000 generations.

For each sub-test an approximation of the Pareto Front corresponding to each multicast group is obtained using a procedure with the following three steps:

1) Each algorithm (MOACS \& MMA) was run ten times to calculate average values.

2) A set solutions " $Y$ " conformed by all solutions of both algorithms was calculated.

3) The dominated solutions were eliminated from " $Y$ ", and an approximation of " $Y_{\text {true }}$ " was created. 


\subsection{Test Problem 1}

The first test problem was the NSF network of Example 1, with ten multicast groups (MG) shown in Table 3.

The number of optimal solutions of the approximated Pareto Front $\boldsymbol{Y}_{\text {true }}$ is presented in Table 4, for each multicast group and load level.

Table 3. Multicast Groups for Test Problem 1.

\begin{tabular}{c|l|c}
\hline MG* & $\multicolumn{1}{|c|}{\mathrm{|}},\left\{\mathrm{N}_{\mathrm{r}}\right\}$ & $\left|\mathrm{N}_{\mathrm{r}}\right|$ \\
\hline 1 & $\{0\},\{1,2,3,4,5,6,7,9,12,13\}$ & 11 \\
2 & $\{12\},\{0,1,2,4,5,6,8,9,11,13\}$ & 10 \\
3 & $\{0\},\{1,2,3,4,5,6,9,12,13\}$ & 9 \\
4 & $\{6\},\{4,8,10,11,12,13\}$ & 6 \\
5 & $\{4\},\{0,1,2,3,6,7,9,10,12,13\}$ & 10 \\
6 & $\{13\},\{0,1,2,3,4,5,6,7,8,9,10,11\}$ & 12 \\
7 & $\{12\},\{0,1,3,5,6,8,9,11,13\}$ & 9 \\
8 & $\{2\},\{0,4,5,7,9,10,12,13\}$ & 8 \\
9 & $\{5\},\{0,4,6,7,8,9,10,11,12\}$ & 9 \\
10 & $\{1\},\{0,7,8,9,12,13\}$ & 6 \\
\hline \multirow{2}{*}{$\mathrm{MG}=$ Multicast Group }
\end{tabular}

Table 4. Number of Optimal Solutions in $Y_{\text {true }}$ for each MG and load level for Test Problem 1.

\begin{tabular}{c|cccc}
\hline \multirow{2}{*}{ MG } & \multicolumn{4}{|c}{$\mid \boldsymbol{Y}_{\text {true }}$} \\
\cline { 2 - 5 } & Low & Half & High & Saturation \\
\hline 1 & 62 & 51 & 62 & 26 \\
2 & 33 & 21 & 32 & 23 \\
3 & 19 & 25 & 25 & 13 \\
4 & 11 & 14 & 10 & 10 \\
5 & 20 & 15 & 9 & 17 \\
6 & 45 & 29 & 31 & 18 \\
7 & 20 & 19 & 13 & 13 \\
8 & 14 & 16 & 14 & 5 \\
9 & 18 & 16 & 19 & 15 \\
10 & 11 & 9 & 13 & 7 \\
\hline
\end{tabular}

\subsection{Test Problem 2}

The second test was carried out using the NTT network topology [7] of Fig. 5, where a delay $d_{i j}$ over each link $(i, j)$ is shown. NTT network consists of 55 nodes and 144 links.

Multicast groups used in this test are shown in Table 5 and the number of optimal solutions of the approximated Pareto Front $Y_{\text {true }}$ is presented in Table 6. 
112 Diego Pinto and Benjamín Barán

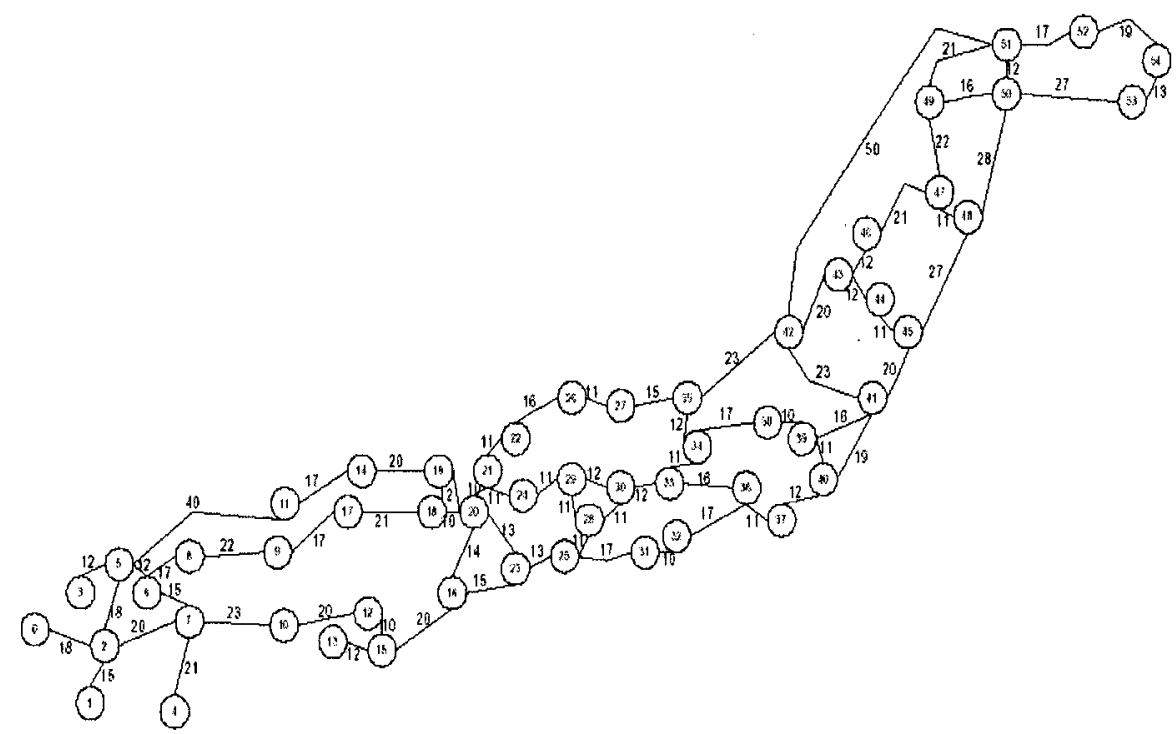

Fig. 5. NTT network used in test problem 2. Numbers over links represent propagation delay in $\mathrm{ms}$.

Table 5. Multicast Groups for Test Problem 2.

\begin{tabular}{|c|c|c|}
\hline $\mathrm{MG}$ & $\{\mathrm{s}\},\left\{\mathrm{N}_{\mathrm{r}}\right\}$ & $\left|N_{r}\right|$ \\
\hline 1 & $\{51\},\{0,3,4,8,13,15,16,22,30,31,40,41,44,47,50,54\}$ & 16 \\
\hline 2 & $\begin{array}{l}\{48\},\{2,3,5,6,7,8,9,10,11,12,14,15,16,20,24,25,28,29,30,31,33,34,40,43,4 \\
4,46,49,50,51,52,54\}\end{array}$ & 31 \\
\hline 3 & $\begin{array}{l}\{46\},\{0,3,5,6,7,12,14,15,16,17,20,23,24,26,28,29,31,32,34,35,37,39,47,48 \\
50\}\end{array}$ & 25 \\
\hline 4 & $\begin{array}{l}\{26\},\{0,1,2,3,4,5,6,7,8,9,10,11,12,13,14,15,16,17,18,19,20,22,23,24,25,27 \\
, 28,29,30,31,32,3334,35,36,37,38,39,40,41,42,43,44,45,46,47,49,50,51,52 \\
, 53,54\}\end{array}$ & 52 \\
\hline 5 & $\begin{array}{l}\{36\},\{1,7,8,12,14,16,18,20,21,25,26,28,30,32,33,34,35,37,39,41,43,44,45, \\
46,48,49,50,51,52,53,54\}\end{array}$ & 31 \\
\hline 6 & $\{30\},\{0,5,10,12,15,25,29,31,36,42,44,46\}$ & 12 \\
\hline 7 & $\{13\},\{4,6,10,11,14,17,18,19,23,28,30,34,37,38,42,44,53\}$ & 17 \\
\hline 8 & $\begin{array}{l}\{21\},\{0,1,3,4,5,6,7,8,9,10,11,12,13,14,15,17,18,19,20,22,23,24,25,26,27,2 \\
8,30,31,32,33,34,35,36,38,39,41,42,43,44,46,47,48,49,50,51,52,53,54\}\end{array}$ & 48 \\
\hline 9 & $\{51\},\{1,3,7,11,15,16,17,18,26,27,30,37,42,43,46,50,52\}$ & 17 \\
\hline 10 & $\begin{array}{l}\{11\},\{1,4,5,6,9,10,12,15,16,17,19,20,22,23,27,29,30,31,32,34,35,36,37,38 \\
, 39,40,42,43,45,4647,48,49,50,51,52,54\}\end{array}$ & 37 \\
\hline
\end{tabular}


Table 6. Numbers of Optimal Solutions $Y_{\text {irue }}$ for each MG and load level, for Test Problem 2.

\begin{tabular}{c|cccc}
\hline \multirow{2}{*}{ MG } & \multicolumn{4}{|c}{$\mid \boldsymbol{Y}_{\text {true }}$} \\
\cline { 2 - 5 } & Low & Half & High & Saturation \\
\hline 1 & 105 & 79 & 35 & 11 \\
2 & 140 & 182 & 130 & 25 \\
3 & 97 & 67 & 58 & 12 \\
4 & 56 & 50 & 27 & 23 \\
5 & 57 & 41 & 26 & 14 \\
6 & 27 & 86 & 23 & 2 \\
7 & 25 & 7 & 11 & 4 \\
8 & 83 & 14 & 17 & 44 \\
9 & 49 & 33 & 9 & 5 \\
10 & 24 & 35 & 80 & 48 \\
\hline
\end{tabular}

\section{Experimental Results}

Next, the experimental results for each test problem and different load levels are presented separately, comparing the results using the proposed MOACS to the corresponding results using MMA.

\subsection{Test Problem 1}

In these tests using the NSF network with 14 nodes and 42 links, it can be easily seen that in general MOACS outperforms MMA finding a larger number of Pareto solutions (see averages in Table 7). Only in column "Average for each $M G$ " for multicast groups $4(42 \%)$ and $9(49.25 \%)$ MMA may seem better than MOACS, but in the rest of the tests, MOACS is widely superior.

Table 7. Comparison of solutions in $Y_{\text {true }}$ for each MG and load level, for Test Problem 1.

\begin{tabular}{|c|c|c|c|c|c|c|c|c|c|c|}
\hline \multirow[t]{2}{*}{ MG } & \multicolumn{2}{|c|}{ Low } & \multicolumn{2}{|c|}{ Medium } & \multicolumn{2}{|c|}{ High } & \multicolumn{2}{|c|}{ Saturation } & \multicolumn{2}{|c|}{$\begin{array}{c}\text { Average for each } \\
M G\end{array}$} \\
\hline & MOACS & MMA & MOACS & MMA & MOACS & MMA & MOACS & MMA & MOACS & MMA \\
\hline 1 & $80 \%$ & $1 \%$ & $96 \%$ & $0 \%$ & $86 \%$ & $0 \%$ & $100 \%$ & $0 \%$ & $90.50 \%$ & $0.25 \%$ \\
\hline 2 & 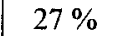 & $1 \%$ & 2 & $21 \%$ & $3 \%$ & $44 \%$ & 10 & $0 \%$ & $\%$ & $24.50 \%$ \\
\hline 3 & $95 \%$ & $1 \%$ & 9 & 0 & $88 \%$ & $2 \%$ & & 0 & 93. & $0.75 \%$ \\
\hline 4 & $36 \%$ & $41 \%$ & $21 \%$ & $43 \%$ & $10 \%$ & $34 \%$ & & $\%$ & $\%$ & $42 \%$ \\
\hline 5 & $\%$ & $2 \%$ & $\%$ & $\%$ & $68 \%$ & $7 \%$ & & $29 \%$ & 73. & $9.75 \%$ \\
\hline 6 & $76 \%$ & $0 \%$ & & $2 \%$ & $87 \%$ & $0 \%$ & & $0 \%$ & $78.25 \%$ & $0.50 \%$ \\
\hline 7 & $\%$ & $28 \%$ & $40 \%$ & $22 \%$ & $15 \%$ & $32 \%$ & 10 & $0 \%$ & $43.75 \%$ & $20.50 \%$ \\
\hline 8 & $100 \%$ & $0 \%$ & $91 \%$ & $4 \%$ & $93 \%$ & $1 \%$ & 100 & $0 \%$ & $96 \%$ & $1.25 \%$ \\
\hline 9 & $28 \%$ & $37 \%$ & $12 \%$ & $76 \%$ & $16 \%$ & $51 \%$ & $65 \%$ & $33 \%$ & $30.25 \%$ & $49.25 \%$ \\
\hline 10 & $55 \%$ & $19 \%$ & $89 \%$ & $11 \%$ & $77 \%$ & $16 \%$ & $57 \%$ & $43 \%$ & $69.50 \%$ & $22.25 \%$ \\
\hline \multicolumn{9}{|c|}{ Average for each Load Level } & \multicolumn{2}{|c|}{ Global Average } \\
\hline & $60 \%$ & $16 \%$ & $62 \%$ & $18 \%$ & $54 \%$ & $19 \%$ & $82 \%$ & $15 \%$ & $64.4 \%$ & $17.1 \%$ \\
\hline
\end{tabular}




\subsection{Test Problem 2}

In this seconds test, MOACS again demonstrates the best performance (see averages in Table 8). See in Table 8 column "Average for each $M G$ ", that only for multicast group $5(15 \%)$ MMA is superior. Also notice that in the "Global Average" MOACS calculated $40 \%$ of $Y_{\text {true }}$ solutions while MMA only found $3 \%$. Even more, considering "Global Average," MOACS achieved $67.2 \%$ of $Y_{\text {true }}$ solutions while MMA only reached $17.1 \%$.

Table 8. Comparison of Solutions with $Y_{\text {true }}$ for each Multicast group and level load for test problem 2

\begin{tabular}{c|rr|rr|rr|rr|rr}
\hline \multirow{2}{*}{ MG } & \multicolumn{2}{|c|}{ Low } & \multicolumn{2}{c|}{ Medium } & \multicolumn{2}{c|}{ High } & \multicolumn{2}{c|}{ Saturation } & \multicolumn{2}{c}{$\begin{array}{c}\text { Average for each } \\
\text { MG }\end{array}$} \\
\cline { 2 - 10 } & MOACS & MMA & MOACS & MMA & MOACS & MMA & MOACS & MMA & MOACS & MMA \\
\hline 1 & $42 \%$ & $5 \%$ & $46 \%$ & $1 \%$ & $59 \%$ & $5 \%$ & $89 \%$ & $0 \%$ & $59 \%$ & $3 \%$ \\
2 & $10 \%$ & $3 \%$ & $10 \%$ & $0 \%$ & $11 \%$ & $0 \%$ & $58 \%$ & $0 \%$ & $22 \%$ & $1 \%$ \\
3 & $27 \%$ & $0 \%$ & $25 \%$ & $0 \%$ & $27 \%$ & $0 \%$ & $65 \%$ & $0 \%$ & $36 \%$ & $0 \%$ \\
4 & $9 \%$ & $1 \%$ & $8 \%$ & $2 \%$ & $10 \%$ & $1 \%$ & $68 \%$ & $0 \%$ & $24 \%$ & $1 \%$ \\
5 & $7 \%$ & $6 \%$ & $2 \%$ & $14 \%$ & $3 \%$ & $7 \%$ & $4 \%$ & $34 \%$ & $4 \%$ & $15 \%$ \\
6 & $96 \%$ & $0 \%$ & $64 \%$ & $0 \%$ & $86 \%$ & $0 \%$ & $100 \%$ & $0 \%$ & $87 \%$ & $0 \%$ \\
7 & $70 \%$ & $1 \%$ & $36 \%$ & $7 \%$ & $60 \%$ & $1 \%$ & $75 \%$ & $25 \%$ & $60 \%$ & $9 \%$ \\
8 & $10 \%$ & $0 \%$ & $10 \%$ & $1 \%$ & $8 \%$ & $2 \%$ & $40 \%$ & $0 \%$ & $17 \%$ & $1 \%$ \\
9 & $64 \%$ & $0 \%$ & $85 \%$ & $0 \%$ & $42 \%$ & $7 \%$ & $100 \%$ & $0 \%$ & $73 \%$ & $2 \%$ \\
10 & $10 \%$ & $0 \%$ & $10 \%$ & $0 \%$ & $9 \%$ & $1 \%$ & $52 \%$ & $0 \%$ & $20 \%$ & $0 \%$ \\
\hline \multicolumn{8}{c|}{ Average for Load Level } \\
\hline
\end{tabular}

It can be concluded from Tables 7 and 8 that MOACS outperforms MMA for this type of MOP, finding a larger number of Pareto solutions.

From these experimental results, the following conjecture can be stated. ACO algorithms as MOACS build relatively good solutions using heuristic information (visibility) and avoiding not feasible solutions; therefore, in general, ACO algorithms are better suited for constructing good solution, compared to Multiobjective Evolutionary Algorithms - MOEAs [11] as MMA. However, the above algorithms are probabilistic; thus, MMA may eventually outperform MOACS, as shown in Table 8 (row 5 and column Average for each $M G$ ).

\section{Conclusions}

This paper introduces a new approach based on MOACS to solve the multicast routing problem. The proposed MOACS is able to optimize simultaneously four objective functions, such as, (1) maximum link utilization $\left(\alpha_{m}\right)$, (2) cost of the routing tree $(C),(3)$ maximum end-to-end delay $\left(D_{m}\right)$ and (4) average delay $\left(D_{a}\right)$. This new proposal is able to solve a multicast routing problem in a truly multiobjective context, considering all four objectives at the same time, for the first time using an algorithm based on Ant Colony Optimization. The new approach calculates not only one possible solution, but a whole set of optimal Pareto solutions 
in only one run. This last feature is especially important since the most adequate solution can be chosen for each particular case without a priori restrictions that may eliminate otherwise good solutions. To validate the new approach, MOACS was compared to the MMA, a representative algorithm for solving the multicast routing problem in a truly multiobjective context, for Traffic Engineering. The experimental results showed that MOACS is able to find a larger number of Pareto solutions than MMA for different network topologies, different load level and various multicast groups, i.e. MOACS found better solutions in average than MMA.

As a future work, the authors will perform more tests over other network topologies and other metrics will be also considered to make sure that algorithms based on Ant Colonies are a promising approach for traffic engineering. At the same time, authors consider the study of convergence time for the proposed algorithm, as well as the control traffic load it causes.

\section{References}

1. A. Tanenbaum, Computer Networks, Prentice Hall $4^{\circ}$ Edition, 2003.

2. Y. Seok, Y. Lee, Y. Choi, and C. Kim, "Explicit multicast routing algorithm for constrained traffic engineering", IEEE $7_{\text {th }}$ International Symposium on Computer and Communications (ISCC'02). Italy, 2002.

3. Y. Donoso, R. Fabregat, and J. Marzo, "Multiobjective optimization algorithm for multicast routing with traffic engineering", IEEE $3^{\text {rd }}$ International Conference on Networking (ICN'2004), Guadalupe, French Caribbean, March - 2004.

4. M. Schaerer, and B. Barán. "A Multiobjective Ant Colony System For Vehicle Routing Problem With Time Windows", IASTED International Conference on Applied Informatics, Innsbruck, Austria, 2003.

5. Y. Liu, and J. Wu. "The degree-constrained multicasting algorithm using ant algorithm" IEEE 10 th International Conference on Telecommunications" 2003.

6. J. Gu, C. Chu, X. Hou, and Q. Gu. "A heuristic ant algorithm for solving QoS multicast routing problem" Evolutionary Computation, 2002. CEC '02. Volume 2, pp 1630-1635.

7. J. Crichigno, and B. Barán. "Multi-objective Multicast Routing Algorithm for Traffic Engineering" ICCCN'2004, California, USA, 2004.

8. E. Zitzler, and L. Thiele, "Multiobjective Evolutionary Algorithms: A comparative Case Study and the Strength Pareto Approach", IEEE Trans. Evolutionary Computation, Volume 3, No. 4, 1999, pp 257-271.

9. D. A. Van Veldhuizen. "Multiobjective Evolutionary Algorithms: Classifications, Analysis, and New Innovations", Ph.D Thesis, Graduated School of Engineering of the Air Force Institute of Technology, Air University, 1999.

10. M. Dorigo, and L. M. Gambardella. "Ant Colony System: A cooperative learning approach to the traveling salesman problem" IEEE Transactions on Evolutionary Computation, 1: 1, pp 53-66, 1997.

11. M. Guntsch and M. Middendorf. "A Population Based Approach for ACO". In Stefano Cagnoni, Jens Gottlieb, Emma Hart, Martin Middendorf, and Günther Raidl, Applications of Evolutionary Computing, Proceedings of EvoWorkshops2002: EvoCOP, EvolASP, EvoSTim, Springer-Verlag ,vol. 2279, pp 71-80, Kinsale, Ireland, 2002. 